\title{
Pillars, Jets and Dynamical Features
}

\section{Matthias Gritschneder ${ }^{1,2}$, Andreas Burkert ${ }^{2,3}$, Thorsten Naab ${ }^{2,4}$, Stefanie Walch ${ }^{5}$}

\author{
${ }^{1}$ Kavli Institute for Astronomy and Astrophysics, Peking University, 100871 Beijing, China \\ email: gritschneder@pku.edu.cn \\ ${ }^{2}$ Universitäts-Sternwarte München, 81679 München, Germany \\ ${ }^{3}$ Max Planck Institut für Extraterrestrische Physik, 85748 Garching bei München, Germany \\ ${ }^{4}$ Max Planck Institut für Astrophysik, 85740 Garching bei München, Germany \\ ${ }^{5}$ School of Physics \& Astronomy, Cardiff University, Cardiff CF24 3AA, United Kingdom
}

\begin{abstract}
We present high resolution simulations on the impact of ionizing radiation on turbulent molecular clouds. The combination of hydrodynamics, gravitational forces and ionization in the tree-SPH code iVINE naturally leads to the formation of elongated filaments and clumps, which are in excellent agreement with the pillars observed around HII regions. Including gravity the formation of a second generation of low-mass stars with surrounding protostellar disks is triggered at the tips of the pillars, as also observed. A parameter study allows us to determine the physical conditions under which irregular structures form and whether they resemble large pillars or a system of small, isolated globules.
\end{abstract}

\section{Introduction}

Stars are known to form in turbulent, cold molecular clouds. When massive stars ignite, their UV-radiation ionizes and heats the surrounding gas, leading to an expanding HII bubble. As soon as the HII region breaks through the surface of the molecular cloud a lowdensity, optically thin hole is formed which reveals the otherwise obscured interior. At the interface between the HII region and the molecular gas peculiar structures, often called pillars, are found. In general, the pillars point like fingers towards the ionizing source (see Figure 1d) and show a common head-to-tail structure. Most of the mass is concentrated in the head which has a bright rim facing the young stars. Thin, elongated pillars connect the head with the main body of the molecular cloud. They have typical widths of $0.1-$ $0.7 \mathrm{pc}$ and are $1-4 \mathrm{pc}$ long (Gahm et al. 2006, Schuller et al. 2006). Observations show that the pillars are not smooth, but built of small substructures, filaments and clumps (Pound 1998). Some filaments run diagonal across the pillars, suggesting a complex twist into a helical structure (Carlqvist et al. 2003). This is also supported by spectroscopic measurements of the line-of-sight (LOS) gas velocity: the pillars show a bulk motion away from the ionizing stellar sources with a superimposed complex shear flow that could be interpreted as corkscrew rotation (Gahm et al. 2006). Occasionally, close to the tip of the head small spherical gas clumps are observed to break off and float into the hot HII region. These so called evaporating gaseous globules (EGGs) have been found with HST e.g. in the Eagle Nebula (McCaughrean \& Andersen 2002). If stars with surrounding gas discs happen to form in these clumps they transform into evaporating proto-planetary discs, so called proplyds. Direct signatures of star formation are also found in the head of the pillars, e.g. through jets from obscured proto-stars piercing through the surface into the HII region (e.g. in Eta Carina, Smith et al. 2000). 
Early models of pillar formation suggested that the pillars form by Rayleigh-Taylor instabilities when the expanding hot, low-density HII region radially accelerates the cold, dense gas (Frieman 1954). This has been ruled out by the observations of the complex flows inside the pillars. Another possibility is the radiation-driven implosion (e.g. Bertoldi 1989, Lefloch \& Lazareff 1994, Miao et al. 2009) of preexisting dense cores. This scenario results in cometary-shaped structures. However, the overall shape of the pillars in M16 would require several cores with fine tuned positions and relative velocities. Especially the veil between the two smaller pillars in Figure 1d poses a real challenge. A third scenario is the collect and collapse model. Here, the radiation sweeps up a large shell, which then fragments to form stars and pillars (e.g. Elmegreen \& Lada 1977, Klein et al. 1980). However, the timescales $(>5 \mathrm{Myr})$ and masses $\left(>1000 M_{\odot}\right)$ involved (Elmegreen et al. 1995, Wünsch \& Palouš 2001) are much larger than in M16. Therefore, this scenario is more likely i.e. in supernova-driven shells.

We demonstrate that the observed pillars form naturally from the ionization of a turbulent molecular cloud, representing substructure that is excavated by the stellar UV-radiation and then compressed by the high-pressure HII environment. This process has already been studied on large scales (Mellema et al. 2006, Dale et al. 2007, Mac Low et al. 2007). We present, for the first time, simulations with a high enough resolution (100 AU spatial and $2 \times 10^{-4} M_{\odot}$ in mass) to follow the formation of individual pillars.

\section{Results}

Because of its non-linearity and high complexity, a deeper insight into the evolution of HII regions and their interaction with the surrounding gas can only be gained with numerical simulations. For this purpose we developed a new parallelized 3-dimensional hydrodynamical code to simulate radiative ionization of a turbulent molecular cloud from a nearby star cluster with so far unprecedented resolution (Gritschneder et al. 2009a, Gritschneder et al. 2009b, Gritschneder et al. 2010). Figure 1(a,b,c,e) shows the results of two typical simulations, corresponding to the ionization of a cloud with a temperature of $T=10 \mathrm{~K}$ and a mean number density of $n=300 \mathrm{~cm}^{-3}$ at turbulent velocities of Mach 5 and 7 , respectively. This is the range of random velocities that are typically measured spectroscopically in molecular clouds. The HII region extends north towards the massive stars that are located outside of the computational domain. Along channels of low gas density, the ionizing radiation penetrates deeply into the molecular cloud, generating channels of hot, pressurized gas. Ionization is however less efficient along line-of sights with a density enhancement near the cloud surface. These regions are the seeds of the pillars. The enhancement is compressed into a clump that becomes the head of the pillar. Heated gas evaporates off the surface and streams into the direction of the ionizing star cluster. Momentum conservation drives a compression wave into the clump, leading to a flattened, optically thick "cap" that casts its shadow radially away from the ionizing source. The gas in the shadow remains cold while the environment is ionized and heated. An isolated, elongated pillar forms that is surrounded by hot and diffuse gas. The structure in the pillar is very clumpy and filamentary with an irregular and twisted velocity field which still traces the previous molecular cloud turbulence.

Figure 1e shows a zoom on the pillars that form in our simulations on the same scale as the HST observation of the pillars of creation (Figure 1d). The size and geometry of the simulations matches very well the observed structures. We also find excellent agreement with respect to kinematics and density substructure. The pillars surface densities in our simulations are $N\left(H_{2}\right) \approx 3 \times 10^{20} \mathrm{~cm}^{-2}$ on average. In total, the mass contained in Figure $1 \mathrm{e}$ is $\approx 45 M_{\odot}$, the most prominent pillar has a mass of $\approx 15 M_{\odot}$, similar to 

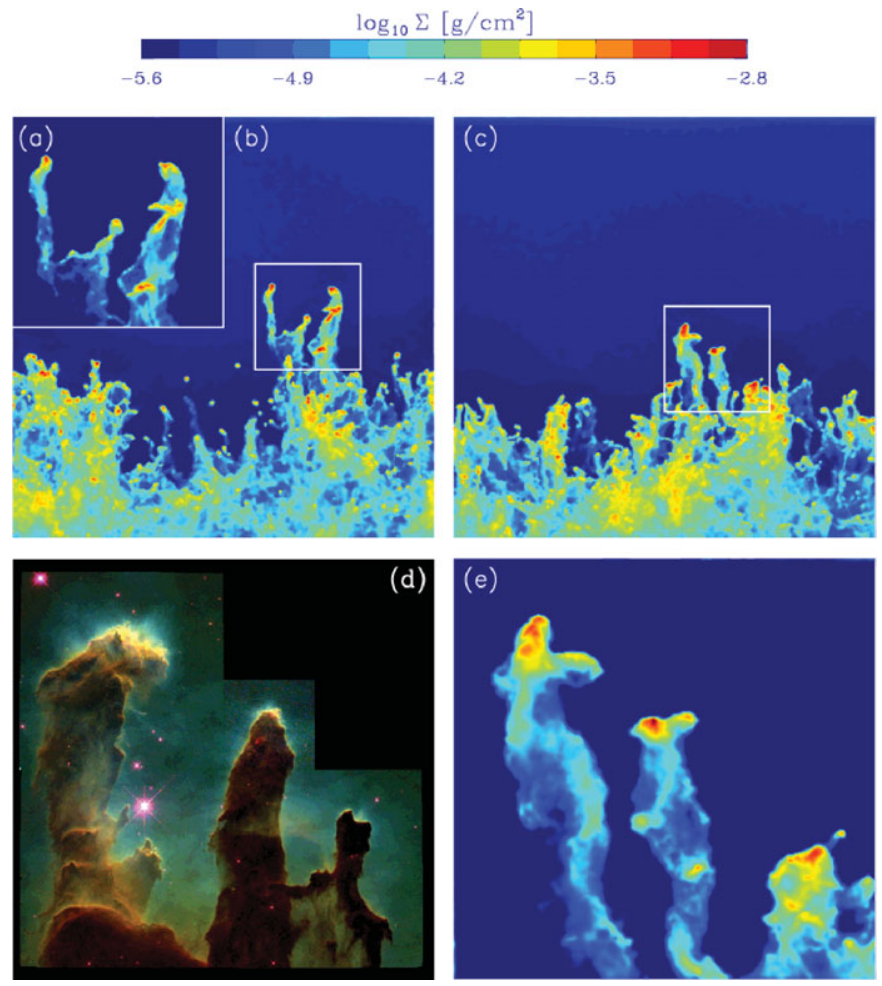

Figure 1. The evolutionary stages of two cloud ionization simulations, $t=500 \mathrm{kyr}$ after the ignition of the ionizing source, are compared with the observed pillars of creation in M16 by Hester et al. 1996. We show colour coded gas surface density, integrated along the z-axis. The upper left (b) and upper right (c) panels correspond to clouds with initial turbulent velocities of Mach 7 and Mach 5, respectively. The box size is $4 \mathrm{pc} \times 4 \mathrm{pc}$. The lower right panel (e) shows a zoom into the upper right picture with a box size of $1 \mathrm{pc} \times 1 \mathrm{pc}$. Here and for the inset of the upper left panel (a) the colour-coding is shifted by an order of magnitude to higher surface densities. The observation in the lower left panel (d) (Hester et al. 1996) has the same physical length scale as the zoomed simulated pillars to the right (e).

the observed 9.2 $M_{\odot}$ in the Dancing Queen trunk in NGC 7822 (Gahm et al. 2006). In addition, the simulated line widths with a standard mean deviation of $\approx 0.38 \mathrm{~km} \mathrm{~s}^{-1}$, corresponding to a FWHM $0.94 \mathrm{~km} \mathrm{~s}^{-1}$, are in excellent agreement with the observed FWHM $1.2 \mathrm{~km} \mathrm{~s}^{-1}$. Furthermore, these lines resemble a cork-screw pattern, as also observed (for details see Gritschneder et al. 2010).

The pillars heads continuously change due to this internal turbulence seen in the linewidths and their streaming velocity. An example is the small spherical gas globule that is about to break off from the tip of the head in the lower right corner of the snapshot shown in Figure 1e. It results from a small sub-clump that separates from the turbulent head due to its relative velocity, drifts into the hot HII region and forms an isolated EGG. The irregular, elongated vertical gas filament in Figure 1a with a veil of cold gas in its shadow results from the head's tangential motion perpendicular to the impinging radiation. A similar structure is observed in M16 (Figure 1d). At the same time the head is pushed by gas evaporation into the pillar, sweeping up additional cold and clumpy gas. The formation and lifetime of a pillar depends strongly on the local velocity field of the parental molecular cloud, prior to ionization. Parameter studies (Gritschneder et al. 2010) show that the turbulent gas velocity of the molecular cloud has to exceed Mach 
numbers $M \sim 2$ at a density of $n=300 \mathrm{~cm}^{-3}$ for substructures to be dense and massive enough in order to survive the ionization and cast long shadows. If the turbulent velocity is on the other hand too large $(M \geqslant 10)$, the tangential velocities are in general high enough to destroy the coherence of forming pillars quickly, leading to larger numbers of isolated globules and rarely to massive structures. We find that the size of the pillars is typically $2.5 \%$ of the wavelength of the dominant mode in the turbulent power-spectrum. Applying these results to the M16 pillars, we conclude that their parental molecular cloud was characterized by irregular motion in the range of $M \approx 5-7$, corresponding to velocities of $1.0-1.4 \mathrm{~km} \mathrm{~s}^{-1}$ with a turbulent driving scale of order $4 \mathrm{pc}$.

\section{Conclusions}

In summary, it is remarkable that the simple model of a turbulent, cold cloud being ionized by a nearby star cluster can reproduce the observed structures around $\mathrm{H}$ II regions so well. For the first time the complex morphology and kinematics can be matched simultaneously. We conclude that ionization, momentum exchange between the evaporating hot gas and the cold surrounding as well as shadowing effects dominate the formation and evolution of structures in ionization fronts around star-forming regions. Large, coherent pillars form only under certain conditions that provide interesting information about the initial molecular cloud environment. Low-mass star formation can be triggered in the dense heads of the pillars, facing the young stellar cluster.

\section{Acknowledgements}

This research was supported financially by the Cluster of Excellence "Origin and Structure of the Universe" which also partly funded the SGI Altix 3700 Bx2 supercomputer where all simulations were performed.

\section{References}

Bertoldi, F. 1989, ApJ, 346, 735

Carlqvist, P., Gahm, G. F., \& Kristen, H. 2003, A\& A, 403, 399

Dale, J. E., Clark, P. C., \& Bonnell, I. A. 2007, MNRAS, 377, 535

Elmegreen, B. G., Kimura, T., \& Tosa, M. 1995, ApJ, 451, 675+

Elmegreen, B. G. \& Lada, C. J. 1977, ApJ, 214, 725

Frieman, E. A. 1954, ApJ, 120, 18

Gahm, G. F., Carlqvist, P., Johansson, L. E. B., \& Nikolić, S. 2006, A\&̛A, 454, 201

Gritschneder, M., Naab, T., Burkert, A., Walch, S., Heitsch, F., \& Wetzstein, M. 2009a, MNRAS, 393,21

Gritschneder, M., Naab, T., Walch, S., Burkert, A., \& Heitsch, F. 2009b, ApJ Letters, 694, L26

Gritschneder, M., Burkert, A., Naab, T., \& Walch, S. 2010, ArXiv e-prints 1009, arXiv:1009.0011

Hester, J. J. et al. 1996, AJ, 111, 2349

Klein, R. I., Sandford, II, M. T., \& Whitaker, R. W. 1980, Space Science Reviews, 27, 275

Lefloch, B. \& Lazareff, B. 1994, A\& A, 289, 559

Mac Low, M.-M., Toraskar, J., Oishi, J. S., \& Abel, T. 2007, ApJ, 668, 980

McCaughrean, M. J. \& Andersen, M. 2002, A\&A, 389, 513

Mellema, G., Arthur, S. J., Henney, W. J., Iliev, I. T., \& Shapiro, P. R. 2006, ApJ, 647, 397

Miao, J., White, G. J., Thompson, M. A., \& Nelson, R. P. 2009, ApJ, 692, 382

Pound, M. W. 1998, ApJ Letters, 493, L113+

Schuller, F., Leurini, S., Hieret, C., Menten, K. M., Philipp, S. D., Güsten, R., Schilke, P., \& Nyman, L. 2006, A\&BA, 454, L87

Smith, N., Egan, M. P., Carey, S., Price, S. D., Morse, J. A., \& Price, P. A. 2000, ApJ Letters, 532, L145

Wünsch, R. \& Palouš, J. 2001, A\&̧A, 374, 746 